\title{
Resolución atómica de elementos ligeros utilizando HAADF y ABF-STEM con corrección de Cs y bajo voltaje* $^{*}$
}

\section{Atomic resolution imaging of light elements using low voltage Cs-corrected HAADF and ABF-STEM}

\author{
Rodrigo Esparza**
}

\begin{abstract}
Scanning transmission electron microscopy (STEM) can give structural and chemical information down to $0.1 \mathrm{~nm}$ of spatial resolution, which sub-angstrom resolution is achieved with a spherical aberration corrector for the probe. In the STEM, the electron probe is focused and scanned over the sample and the image is formed by measuring the transmitted electron intensity arising after the electron-specimen interactions. The scattered electron intensity that cross the sample can be employed to obtain bright and dark field images. The STEM is a powerful instrument to understand the physical properties of nanostructures which requires the local structure and local chemistry to be determined at the atomic scale. Therefore, STEM is a technique able to identify the atomic columns and the position of atoms using simulation techniques. In this work, the basic instrumental parameters using high angle annular dark field (HAADF-STEM) and annular bright field (ABF-STEM) modes were evaluated using a standard sample of Si[110]. Also, experimental HAADF-STEM and annular bright field (ABF-STEM) images of $\mathrm{LaAlO}_{3}$ sample were obtained at $80 \mathrm{kV}$ and compared with simulated images obtained by using the multi slice dynamical diffraction method. Using the ABF-STEM technique, it was possible to clearly visualize the atomic columns of the La, $\mathrm{O}$ and Al elements included in the sample. Images simulated were found to agree well with experimental images.
\end{abstract}

KEYWORDS: scanning transmission electron microscopy, annular bright field, high angle annular dark field, image simulations.

RESUMEN: La microscopía electrónica de barrido y transmisión (STEM) puede ofrecer información estructural y química con resolución espacial del orden de $0.1 \mathrm{~nm}$, es posible alcanzar tal resolución utilizando un corrector de aberración esférica para el haz de prueba. En el STEM, un haz de electrones es enfocado y escaneado sobre la muestra, por lo que la imagen se forma midiendo la intensidad electrónica transmitida que surge después de las interacciones electrón-muestra. La intensidad de los electrones dispersados que atraviesan la muestra puede ser empleada para obtener imágenes de campo claro y campo obscuro. El microscopio STEM es un poderoso instrumento para estudiar las propiedades físicas de las nanoestructuras, que requieren de un análisis estructural y químico a nivel atómico. Por lo tanto, STEM es una técnica capaz de identificar columnas atómicas y la posición de los átomos auxiliándose con técnicas de simulación. En este trabajo, los parámetros instrumentales básicos del microscopio en modo campo

* R. Esparza agradece al Kleberg Advanced Microscopy Center de la UTSA por sus instalaciones.

** Universidad Nacional Autónoma de México, Centro de Física Aplicada y Tecnología Avanzada, Boulevard Juriquilla 3001, Santiago de Querétaro, Qro., 76230, México.

Correo electrónico: resparza@fata.unam.mx 
Mundo Nano | ARTículos DE INVESTIGACIóN | www.mundonano.unam.mx

13(25), 45-60, julio-diciembre 2020 | https://doi.org/10.22201/ceiich.24485691e.2020.25.69636

Rodrigo Esparza

oscuro anular de gran ángulo (HAADF-STEM) y campo claro (BF-STEM) fueron evaluados utilizando una muestra estándar de Si[110]. Además, imágenes experimentales de HAADF-STEM y campo claro anular (ABF-STEM) de una muestra de $\mathrm{LaAlO}_{3}$ se consiguieron a $80 \mathrm{kV}$ de operación y comparadas con imágenes simuladas obtenidas con el método de multicapas, el cual se basa en la teoría dinámica de la difracción. Mediante la técnica ABF-STEM fue posible visualizar claramente las columnas atómicas de los elementos La, $\mathrm{O}$ y Al presentes en la muestra. Se encontró que las imágenes simuladas coinciden bien con las imágenes experimentales.

PALABRAS CLAVE: microscopía electrónica de transmisión/barrido, campo oscuro anular de alto ángulo, campo claro anular, simulación de imágenes.

\section{Introducción}

El coeficiente de aberración esférica $(\mathrm{Cs})$ ha sido el principal factor que restringe la resolución en el campo de la microscopía electrónica de transmisión (TEM). Sin embargo, la implementación del corrector de Cs en las últimas décadas ha incrementado el desarrollo de los microscopios electrónicos, alcanzando imágenes con una resolución por debajo de $0.1 \mathrm{~nm}$. Aun más, los beneficios de la microscopía electrónica de barrido en transmisión (STEM) han sido mayores que los del TEM convencional, debido a la mejora en la relación señal/ruido, esto es posible gracias a la disponibilidad de un haz con mayor corriente (Haider et al., 1998). Adicionalmente, las imágenes con resolución atómica en modo STEM de campo oscuro de gran ángulo presentan la gran ventaja de ser directamente interpretables, a diferencia de aquellas en modo TEM, en las que es necesaria su comparación con imágenes simuladas para conseguir una adecuada interpretación, debido a la influencia de los factores propios del sistema óptico-electrónico de un TEM (función de transferencia de contraste).

Un STEM es un microscopio donde los electrones pasan a través de una muestra delgada, al igual que en un TEM. Sin embargo, el STEM se diferencia del TEM convencional por enfocar el haz de electrones en un punto que escanea la muestra, similar al microscopio electrónico de barrido (SEM). Usando el STEM y un detector anular de gran ángulo, es posible formar imágenes donde el contraste de estas está directamente relacionado con el número atómico de la muestra (Crewe, Wall y Langmore, 1970).

El STEM también ofrece importantes beneficios en imágenes en el modo campo oscuro anular de gran ángulo (HAADF-STEM). En este modo, el ángulo interno del detector anular se hace tan grande que no se recogen electrones difractados de Bragg. Las imágenes, por lo tanto, provienen de electrones dispersados incoherentemente que han pasado muy cerca de los núcleos atómicos de la muestra. Por consiguiente, imágenes con alta resolución son posibles sin contraste de difracción, el cual es no deseado dado que puede enmascarar información estructural. La señal de HAADF-STEM es directamente proporcional a la densidad y/o espesor de la muestra y proporcional a $Z^{\mathrm{n}}$, donde $\mathrm{Z}$ es el número atómico de los elementos de la muestra y el exponente $n$ es cercano a 1.7. De este modo, es posible producir imágenes 
que muestren contraste debido a la combinación del espesor másico, donde la intensidad es proporcional al número de átomos e imágenes de contraste $\mathrm{Z}$, donde la señal es proporcional al número atómico promedio de la muestra (Pennycook et al., 1996). HAADF-STEM es adecuado para muestras inorgánicas y orgánicas, así como para materiales cristalinos y amorfos.

En el pasado, se favoreció el uso del TEM con altos voltajes porque en esas condiciones de operación se reducen los efectos de aberración cromática $(\mathrm{Cc})$ y aumenta la resolución atómica debido a la disminución de la longitud de onda (Dupouy, 2017), sin embargo, el desarrollo de correctores de aberración esférica $(C s)$ ha permitido la resolución atómica a voltajes de operación más bajos (Bell, Russo y Kolmykov, 2012). Actualmente, el STEM utilizando bajos voltajes es de gran interés porque produce menos daño durante las observaciones, principalmente en materiales sensibles al haz de electrones. No obstante, la reducción del voltaje de aceleración requiere aumentar el semiángulo de convergencia para formar un haz de electrones fino, e incrementa la dispersión inelástica de los electrones. Por lo tanto, es necesario compensar las aberraciones geométricas hasta semiángulos de convergencia más altos cuando se usan voltajes de aceleración bajos (Sasaki et al., 2010). La incorporación de correctores de aberración, en particular la instalación exitosa del corrector de Cs para el haz de prueba en la columna del STEM, ha permitido aumentar la resolución hasta niveles sub-Angstrom (Spence, 2013; Hawkes, 2004). Al incorporar el corrector en la columna óptica del microscopio, después de las lentes condensadoras, se tiene un STEM corregido por aberración que ha demostrado ser una herramienta versátil y poderosa para analizar nanomateriales avanzados a escala atómica (Esparza et al., 2013; Mayoral et al., 2011).

Como ejemplo de las aplicaciones del STEM, podemos mencionar la caracterización estructural de nanopartículas bimetálicas, tanto con una estructura del tipo núcleo-coraza como aleación. La morfología y estructura de las nanopartículas bimetálicas de AuPd se ha estudiado mediante la técnica de HAADF-STEM de alta resolución, donde las imágenes mostraron nanopartículas de Au con superficies enriquecidas con átomos de Pd (Esparza et al., 2014). Mientras que en nanopartículas bimetálicas de PdPt con una estructura del tipo aleación se ha podido determinar, con la ayuda de métodos de simulación, la cantidad tanto de átomos de Pd como de Pt por columna atómica (Rodríguez-Proenza et al., 2018). Asimismo, podemos mencionar imágenes de HAADF-STEM obtenidas de láminas de $\mathrm{MoS}_{2}$ donde el efecto del contraste- $Z$ es notable, debido a la diferencia en número atómico de los elementos Mo y S, consiguiendo un contraste débil para las columnas atómicas del S y un mayor contraste para las columnas de Mo (Garcia et al., 2014). Utilizando las imágenes experimentales, así como imágenes obtenidas por simulación, se realizó un análisis cuantitativo a nivel atómico para determinar el número de capas de $\mathrm{MoS}_{2}$.

Por otro lado, las imágenes obtenidas de campo claro anular $(\mathrm{ABF})$ en STEM ha estimulado el interés de la investigación en los últimos años debido 
a su capacidad de una visualización directa de elementos ligeros (Zhou et al., 2016; Ishikawa et al., 2011; Okunishi et al., 2009). La transferencia de contraste en ABF-STEM proporciona una mejor resolución, así como una transferencia de contraste no oscilante que el campo claro (BF)-STEM convencional, lo cual permite que las imágenes sean fácilmente interpretables a diferencia de BF-STEM (Okunishi et al., 2009). Esto ha permitido identificar claramente átomos individuales; así, es importante una cuantificación de las imágenes mediante simulaciones teóricas, necesarias para una interpretación correcta de la imagen, porque la intensidad de la columna atómica depende en gran medida de la cantidad de desenfoque y el grosor de la muestra. Sin embargo, la simulación de imágenes de STEM presenta un desafío debido al tiempo requerido para generar datos para cada posición del haz de electrones (Dwyer, 2010).

El trabajo realizado en esta investigación está relacionado con la evaluación del rendimiento del instrumento STEM equipado con un corrector de $\mathrm{Cs}$, para lo cual se utilizan muestras cristalinas de silicio $(\mathrm{Si})$ orientadas a lo largo del eje de zona [110]. El STEM con aberración esférica corregida también se usó en la caracterización estructural de muestras de aluminato de lantano $\left(\mathrm{LaAlO}_{3}\right)$ para la identificación de los elementos presentes en la muestra. Se llevaron a cabo comparaciones entre imágenes HAADF-STEM y ABF-STEM, así como simulaciones teóricas de imágenes de las muestras cristalinas.

\section{Procedimiento experimental}

Las condiciones instrumentales del microscopio electrónico Jeol JEM-ARM200F con un cañón de emisión de campo tipo Schottky se muestran en la tabla 1. El voltaje de aceleración fue de $80 \mathrm{kV}$ y una longitud de cámara de 80 $\mathrm{mm}$. El microscopio está equipado con un corrector de aberración esférica (Cs) CEOS en el sistema de iluminación. El coeficiente de aberración esférica corregido es de aproximadamente $1 \mu \mathrm{m}$. Los ángulos de recolección para las técnicas de imagen HAADF y ABF se ajustaron en el rango de 90-170 mrad y 11-22 mrad, respectivamente. Además, se obtuvieron imágenes HAADFSTEM y BF-STEM de una muestra estándar de Si orientada a lo largo de la dirección [110], así como de una muestra de $\mathrm{LaAlO}_{3}$ (LAO).

La preparación de las muestras de $\operatorname{Si}[110]$ y LAO se realizó mediante el uso de un microscopio de doble haz (FIB). El procedimiento general para la preparación de las muestras consta de cuatro etapas: depósito de una capa de platino, desbaste en las secciones laterales a la capa de platino, corte y extracción del material (lamela) el cual se pega a una rejilla de $\mathrm{Cu}$, finalmente se adelgaza la muestra con un espesor menor a de $50 \mathrm{~nm}$.

Los coeficientes de las aberraciones del STEM se midieron a $80 \mathrm{kV}$ y fueron obtenidos después de la alineación del corrector mediante el programa del corrector CEOS (tabla 1). Estos valores se consideraron para la si- 
mulación de las imágenes y el ronchigrama. Las simulaciones de las imágenes HAADF, BF y ABF-STEM se realizaron utilizando el programa QSTEM (Koch, 2002) que utiliza el algoritmo modificado del método de multicapas (Kirkland, 1987) basado en la teoría dinámica de $n$ haces de Cowley y Moodie (Cowley y Moodie, 1957). Para simular el ronchigrama se utilizó el programa Dr. Probe Light (Barthel, 2007). Este programa es aconsejable para interpretar imágenes de STEM que ayudan a comprender los efectos de las aberraciones y a optimizar el ronchigrama mediante la simulación avanzada del haz de electrones.

Tabla 1. Valores de los coeficientes obtenidos para el STEM medidos a 80 kV, notación de Haider.

\begin{tabular}{ll}
\hline Coeficientes & \\
\hline Desenfoque $C_{1}$ & $-2.503 \mathrm{~nm}$ \\
\hline Astigmatismo 2do. orden $A_{1}$ & $1.722 \mathrm{~nm}$ \\
\hline Coma $B_{2}$ & $39.93 \mathrm{~nm}$ \\
\hline Astigmatismo 3er. orden $A_{2}$ & $77.01 \mathrm{~nm}$ \\
\hline Aberración esférica $C_{3}$ & $0.743 \mu \mathrm{m}$ \\
\hline Aberración Star $S_{3}$ & $67.29 \mathrm{~nm}$ \\
\hline Astigmatismo 4to orden $A_{3}$ & $1.506 \mu \mathrm{m}$ \\
\hline Coma 5to orden $B_{4}$ & $22.23 \mu \mathrm{m}$ \\
\hline Astigmatismo 5to orden $A_{4}$ & $15.45 \mu \mathrm{m}$ \\
\hline Aberración esférica 5to orden $C_{5}$ & $-185.1 \mu \mathrm{m}$ \\
\hline Astigmatismo 6to orden $A_{5}$ & $1.165 \mathrm{~nm}$ \\
\hline
\end{tabular}

Fuente: Haider, Uhlemann y Zach (2000).

\section{Resultados}

Actualmente, la disponibilidad de los correctores de aberración para microscopios electrónicos de barrido en transmisión (STEM) es bastante amplia, aunque todavía son relativamente costosos $(\approx 0.5$ millones de US $\$)$. Estos instrumentos proporcionan una resolución espacial que se acerca a menos de $0.1 \mathrm{~nm}$. Los correctores de aberración producen haces de electrones más pequeños y brillantes que permiten incluso obtener imágenes de un solo átomo (Mishra et al., 2017). La resolución espacial y la corriente del haz tienen como consecuencia directa el mejoramiento del instrumento. Sin embargo, los microscopios con aberración corregida son más sensibles a las vibraciones mecánicas, perturbaciones acústicas y electromagnéticas, así como a los flujos de aire. Por lo que la evaluación del rendimiento del instrumento es una práctica recomendada, particularmente en microscopios electrónicos nuevos. 
La ruta más efectiva para alinear un STEM es mediante el uso del ronchigrama (Haider et al., 2009), el cual se puede describir en óptica como la imagen proyectada o la sombra de la imagen (shadow image) de una muestra formada en el plano de difracción por un haz de electrones convergente enfocado cerca de la muestra. Aunque este método ha sido utilizado durante muchos años por la comunidad interesada en las técnicas del STEM, no es muy socorrido por la comunidad que realiza microscopía electrónica de transmisión convencional (TEM) (Rodenburg y Macak, 2002). El uso del ronchigrama como parte de los procedimientos de alineación es fundamental en la alineación del corrector de aberración esférica disponible para un STEM, al permitirnos determinar las características ópticas (cantidad de aberración) del haz de electrones formado cerca de la muestra. Esto se debe a que la intensidad, formada en el plano de difracción Fraunhofer del microscopio, varía considerablemente con el ángulo, y esta variación es una función muy sensible de las aberraciones de la lente y el desenfoque (Cowley, 1986).

La figura 1a muestra el ronchigrama experimental observado de una película de carbono amorfo obtenido a $80 \mathrm{kV}$ y un coeficiente de aberración esférica Cs de $0.743 \mu \mathrm{m}$. Cabe mencionar que el carbono amorfo proviene de la membrana de soporte de una rejilla de Cu utilizada en microscopía de transmisión. El ronchigrama muestra un área plana coherente superior a 40 mrad en el ángulo medio, correspondiente al ángulo de convergencia de la fase uniforme (Sawada et al., 2008), recordando que para compensar las aberraciones geométricas es necesario ángulos de convergencia más altos cuando se usan voltajes de aceleración bajos, el área plana no tiene una simetría totalmente esférica sino una forma similar a un eje 6 de simetría característico de un corrector Cs tipo hexapolo, en el que seis polos N/S alternan en torno al eje óptico; además del ronchigrama, queda clara el área plana libre de aberraciones. La presencia de aberraciones de orden inferior (véase la tabla 1) se hacen visibles en la parte central del ronchigrama y, por lo tanto, pueden corregirse. Esta área plana es más grande que las obtenidas en microscopios sin corrector Cs y también cuando el haz de electrones no está corregido o está desalineado. Cuando la excitación de cada componente del sistema de iluminación cambia ligeramente, se observan desalineaciones muy pequeñas en el ronchigrama, las cuales cambian severamente su simetría circular. Además, la presencia o ausencia de franjas de interferencia en el ronchigrama indica la cantidad de ampliación incoherente del haz debido a inestabilidades y al efecto del tamaño de la fuente.

La simulación del ronchigrama debe verse como una herramienta esencial para la comunidad que trabaja con STEM con aberración corregida. La figura $1 \mathrm{~b}$ muestra el ronchigrama simulado usando las mismas condiciones de operación del microscopio (véase la tabla 1). Como se puede observar, el ángulo medio es del mismo tamaño que el ronchigrama experimental, además, no se observan franjas de interferencia en la región central del disco transmitido. Estas condiciones ópticas se utilizaron posteriormente en las simulaciones teóricas de las imágenes de STEM. Las figuras 1c y d muestran los ronchi- 
gramas simulados utilizando como valor del coeficiente de aberración esférica $500 \mu \mathrm{m}$ y $1 \mathrm{~mm}$, respectivamente. Como se puede apreciar, en condiciones donde la aberración esférica no está corregida, no existe un área plana coherente. Es importante mencionar que una vez que se alinea el haz de electrones, se coloca una apertura de la lente condensadora en el centro del ronchigrama para obtener un haz de electrones fino y con alta intensidad. Utilizando el STEM con el corrector de Cs, se pueden obtener imágenes simultáneas con alta resolución de las posiciones de los átomos pesados y ligeros, incluso en condiciones de contraste Z (Haider et al., 1998). Algunos parámetros fuertemente relacionados con la forma de la distribución de intensidad del haz de electrones para medir el ancho a la altura media (FWHM) son: el desenfoque, el ángulo de convergencia, la aberración esférica y el nivel de cualquier ampliación incoherente del haz de electrones (James y Browning, 1999). Después de la corrección de la aberración esférica, el microscopio utilizado es capaz de proporcionar un haz de electrones con un FWHM de $0.15 \mathrm{~nm}$ (figura 1e).

Figura 1. a) Ronchigrama de una película de carbono amorfo obtenida a $80 \mathrm{kV}$ y coeficiente de aberración esférica $\mathrm{Cs}=0.743 \mu \mathrm{m} ; \mathrm{b}$ ) ronchigrama simulado usando los parámetros del instrumento; $\mathrm{c}$ ) $\mathrm{Cs}=$ $500 \mu \mathrm{m}$; d) $1 \mathrm{~mm}, \mathrm{y}, \mathrm{e})$ haz de electrones simulado con los coeficientes de aberración medidos durante el experimento, el haz de electrones tiene un FWHM de $0.15 \mathrm{~nm}$.
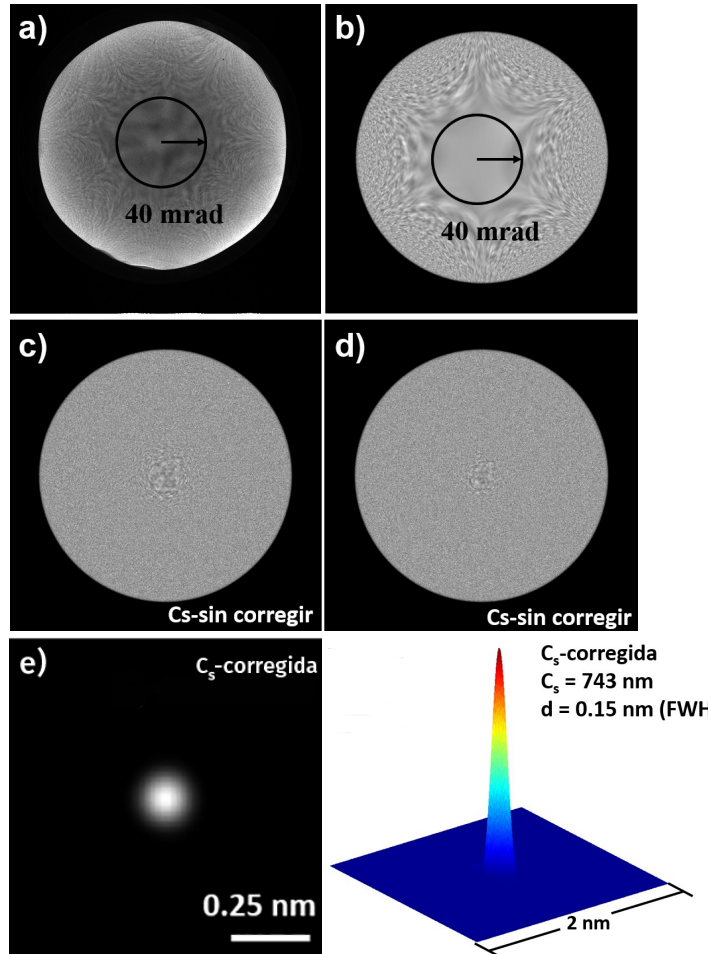

Fuente: Figura a) Cortesía del Kleberg Advanced Microscopy Center; b)-e) elaboración del autor. 
Con el fin de evaluar las condiciones experimentales del microscopio, se obtuvieron imágenes de campo oscuro anular de alto ángulo (HAADF)-STEM y de campo claro (BF)-STEM de estándares de $\mathrm{Si}$ a lo largo de la dirección [110]. Las figuras 2a y 2b muestran las imágenes de HAADF-STEM y BFSTEM obtenidas de la muestra de Si orientada a lo largo de la dirección [110]. En las imágenes, se incluye un recuadro relacionado con la simulación teórica de cada imagen. La simulación de las imágenes se basa en el método multicapas de la teoría dinámica de la difracción de electrones (Koch, 2002). En las figuras también se muestra la transformación rápida de Fourier (FFT) obtenida de cada imagen. Ambas imágenes exhiben las columnas atómicas del Si claramente resueltas con una distancia atómica entre ellas de $0.136 \mathrm{~nm}$. Las imágenes aparecen libres de cualquier distorsión importante. El contraste de las imágenes simuladas de STEM (recuadros), que se basa en los parámetros instrumentales, enumerados en la tabla 1, es muy similar al contraste de las imágenes experimentales. Es importante mencionar que la comparación entre las imágenes experimental y simulada puede dar información sobre la cuantificación atómica y el espesor de la muestra, pero es necesario considerar más parámetros como, por ejemplo, la eficiencia del detector anular (Jones et al., 2014). No obstante, como es conocido, la preparación de la muestra es muy importante en la microscopía electrónica. En el caso particular del STEM con aberración corregida, el grosor de las muestras debe ser menor que el espesor típico de las muestras TEM. Esto se debe a que cuando aumenta el espesor de la muestra, la señal de los electrones dispersados por la muestra se atenúa antes de la detección, debido a eventos múltiples de dispersión elásticos e inelásticos a medida que los electrones viajan a través del substrato, lo cual hace que en el caso de la imagen HAADF-STEM sea muy sensible al espesor de la muestra (Benetatos et al., 2005). El resultado es una disminución de la relación señal/ruido que reduce significativamente la calidad de la imagen. Las FFT de las imágenes muestran una resolución espacial de 0.09 $\mathrm{nm}$. Esto se ilustra con la presencia de la reflexión (4-40). Además, reflexiones correspondientes a las periodicidades del plano (002), que en la estructura espacial real están cinemáticamente prohibidas, se pueden observar en las FFT (Geuens y Van Dyck, 2003). Estas reflexiones, que deberían estar extintas, son débilmente visibles debido a la dispersión múltiple, confirmando aún más que el tamaño del haz es menor de $0.15 \mathrm{~nm}$. Además de estos puntos, en la FFT se pueden observar una gran cantidad de reflejos a una frecuencia espacial muy alta en la dirección vertical. Estos puntos no corresponden a ninguna resolución espacial en la imagen, sino al ruido del barrido del haz e inestabilidades presentes en la muestra (Klie y Zhu, 2005).

Campo claro anular en STEM (ABF-STEM) es una técnica de imagen propuesta hace décadas y utilizada cada vez con mayor frecuencia. La técnica consiste en un detector de campo claro (BF-STEM) en el que el área central queda obstruida - puede hacerse con el bloqueador del haz (beam stopper) del microscopio-, con la finalidad de producir imágenes que muestran co- 
Figura 2. a) HAADF-STEM y b) BF-STEM imágenes de Si[110] tomadas a $80 \mathrm{kV}$, las imágenes muestran una resolución espacial de $0.09 \mathrm{~nm}$.
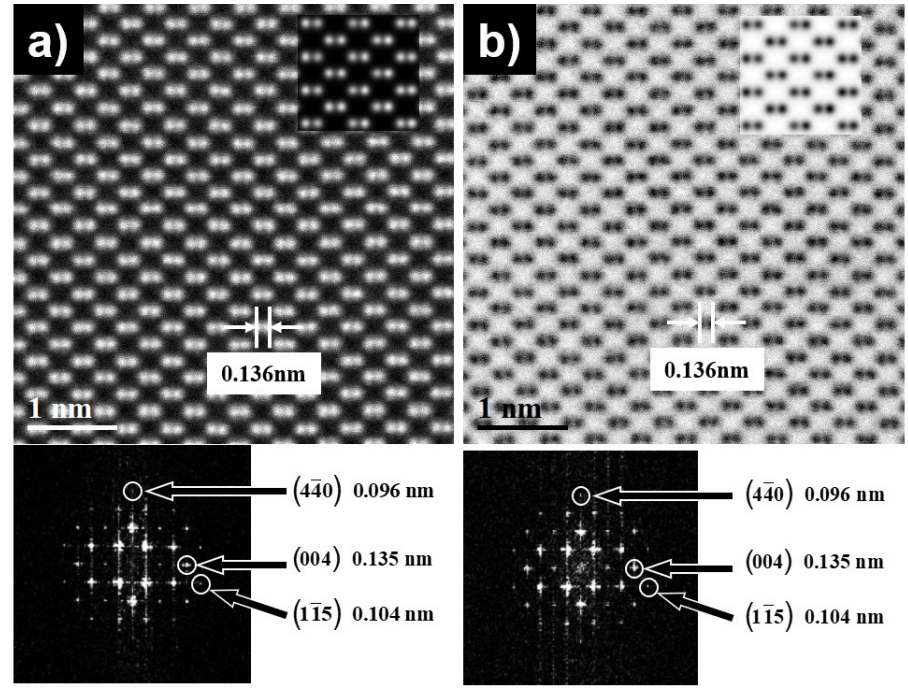

Fuente: Imágenes cortesía del Kleberg Advanced Microscopy Center; recuadros y FFT, elaboración del autor.

lumnas de elementos ligeros y pesados (Findlay et al., 2009). La figura 3a muestra la configuración esquemática donde el detector de HAADF es anular y se coloca por encima de la pantalla de visualización, y el detector BF se coloca debajo de la apertura limitadora de ángulo externo (apertura BF, figura $3 c)$. La figura $3 \mathrm{~b}$ muestra la misma configuración, pero en este caso un disco bloqueador para la formación del ángulo interno es introducido (bloqueador del haz) formando la imagen ABF-STEM. Ambas imágenes HAADF-STEM y ABF-STEM se pueden adquirir simultáneamente usando esta configuración. El semiángulo de la apertura para la formación del haz de electrones se denota por $\alpha=40 \mathrm{mrad}$. Los ángulos interno y externo del detector anular están dados por $\beta_{1}$ y $\beta_{2}$. Para HAADF, $\beta_{1}=90 \mathrm{mrad}$ y $\beta_{2}=170 \mathrm{mrad}$, y para $\mathrm{ABF}, \beta_{1}=11 \mathrm{mrad}$ y $\beta_{2}=22 \mathrm{mrad}$. Punto interesante es el hecho de que el ángulo externo es dos veces mayor que el ángulo interno $\left(\beta_{2} \approx 2 \beta_{1}\right)$, lo que se puede observar en la figura $3 \mathrm{~d}$, donde la relación de los diámetros de los discos es $d_{2} \approx 2 d_{1}$.

Para comprobar la configuración anterior, las figuras $4 \mathrm{a}$ y $\mathrm{b}$ muestran las imágenes experimentales de HAADF-STEM y ABF-STEM, respectivamente, de la fase $\mathrm{LaAlO}_{3}$ (LAO) que tiene una estructura cúbica y un grupo espacial Pm-3m (221), con lantano (La) en las esquinas, aluminio (Al) en el centro y tres átomos de oxígeno $(\mathrm{O})$ en los centros de las caras. A partir de las imágenes experimentales, se obtuvieron distancias interplanares de 0.382, $0.268,0.191$ y $0.171 \mathrm{~nm}$. Dichas distancias interplanares corresponden a los 
Mundo Nano | ARTículos DE INVESTIGACIÓN | www.mundonano.unam.mx

13(25), 45-60, julio-diciembre 2020 | https://doi.org/10.22201/ceiich.24485691e.2020.25.69636

Rodrigo Esparza

Figura 3. a) Configuración del STEM; b) configuración para obtener imágenes ABF-STEM; c) ronchigrama con las aperturas de campo claro y el bloqueador del haz, y, d) detalle de la apertura de campo claro y el bloqueador del haz.
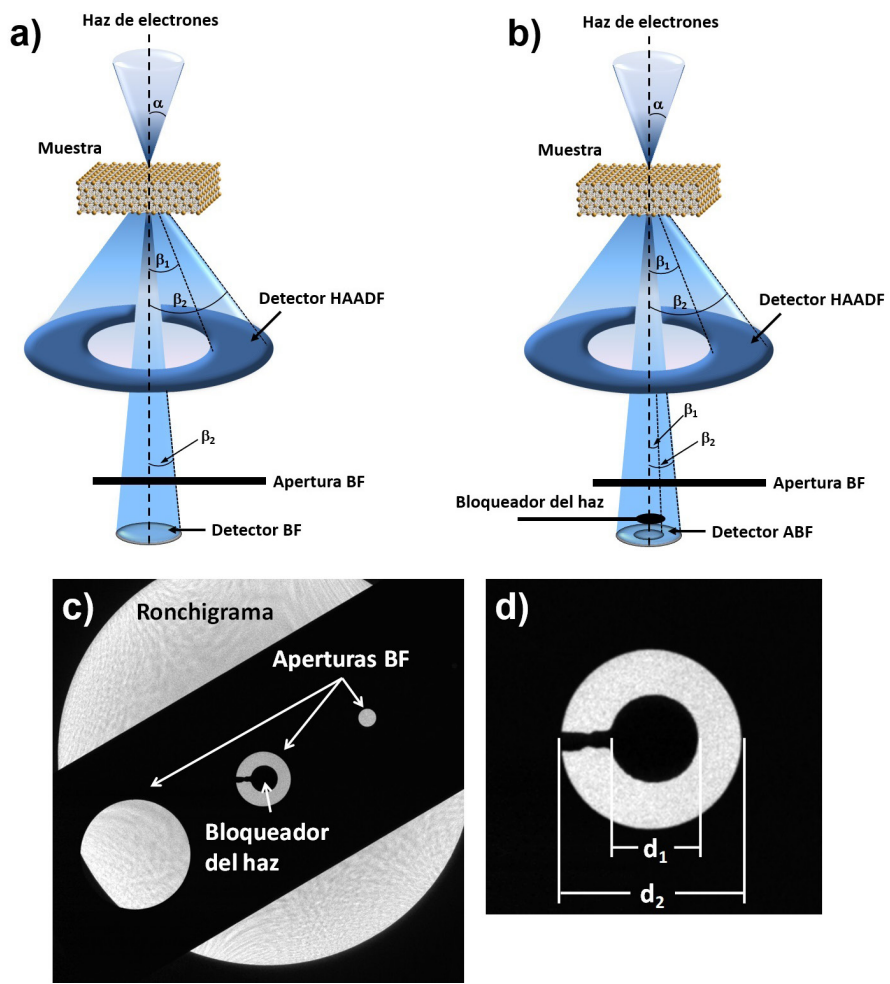

Fuente: a) y b) adaptado de (Kotaka, 2012); c) y d) elaboración del autor.

planos cristalinos (001), (011), (002) y (012), respectivamente, de la estructura del LAO con $\mathrm{a}_{0}=0.3812 \mathrm{~nm}$, y orientada en el eje de zona [001] (ICDD JCPDF 01-070-4123) (Lee et al., 2016).

La imagen de HAADF-STEM muestra una secuencia de puntos muy brillantes, estos puntos brillantes corresponden a la contribución de las columnas de La, dado que La tiene un número atómico mayor que $\mathrm{Al}$ y O (57, 13 y 8 , respectivamente), de modo que la contribución de dispersión de los átomos de $\mathrm{Al}$ y $\mathrm{O}$ es muy débil. Imágenes de HAADF-STEM obtenidas de interfaces de $\mathrm{LaAlO}_{3} / \mathrm{Si}$ y $\mathrm{BiFeO}_{3} / \mathrm{LaAlO}_{3}$ han sido reportadas previamente, donde la intensidad de las columnas Al-O de la fase LAO aparecen débiles en comparación con las columnas de La, como lo mostrado en este trabajo, así como también que las columnas de $\mathrm{O}$ no contribuyen significativamente al contraste de la imagen (Klenov et al., 2005; Huang et al., 2014). De la misma manera, en imágenes de HR-TEM de la fase LAO, esencialmente solo son visibles las columnas atómicas de Al-O (Maurice et al., 2006). Sin embargo, 
como se mencionó anteriormente, las imágenes ABF-STEM proporcionan una señal en la que se pueden analizar claramente tanto columnas de elementos ligeros como pesados con buena relación señal/ruido. En la figura $4 \mathrm{~b}$ se distinguen los átomos de $\mathrm{La}, \mathrm{Al}$ y $\mathrm{O}$, el contraste medio corresponde al $\mathrm{Al}$ y las columnas $\mathrm{O}$ son visibles como puntos con un contraste menor, mientras que los puntos con mayor brillantez corresponden a los átomos de La.

Para comparar la intensidad de las diferentes columnas atómicas, se obtuvieron perfiles de intensidad de ambas imágenes. La figura $4 \mathrm{c}$ muestra los perfiles de intensidad realizados a lo largo de las líneas indicadas en las figuras $4 \mathrm{a}$ y b. A partir de los perfiles de intensidad, está claro que hay picos con diferente intensidad, lo cual refleja el contraste $Z$ de las columnas atómicas. El perfil de intensidad etiquetado con el número 1 es obtenido de la imagen HAADF-STEM, este perfil solo muestra la contribución de los átomos de La como se esperaba. Los perfiles etiquetados como números $2 \mathrm{y}$ 3 se obtuvieron de la imagen ABF-STEM, por lo tanto, se pueden observar elementos ligeros como el $\mathrm{Al}$ y O, así como el La. El perfil etiquetado con el número 2 muestra la contribución de La, que son los picos de mayor intensidad, y también se muestran algunos picos con baja intensidad, que están asociados con $\mathrm{O}$. Es interesante observar en este perfil de intensidad las columnas atómicas de $\mathrm{O}$, que en la imagen de HAADF-STEM no se apreciaban. El perfil de intensidad etiquetado con el número 3 muestra claramente la

Figura 4. a) y b) imágenes de HAADF y ABF-STEM de la fase $\mathrm{LaAlO}_{3}$ a lo largo del eje de zona [001]; c) diferentes perfiles de intensidad obtenidos de las imágenes STEM.
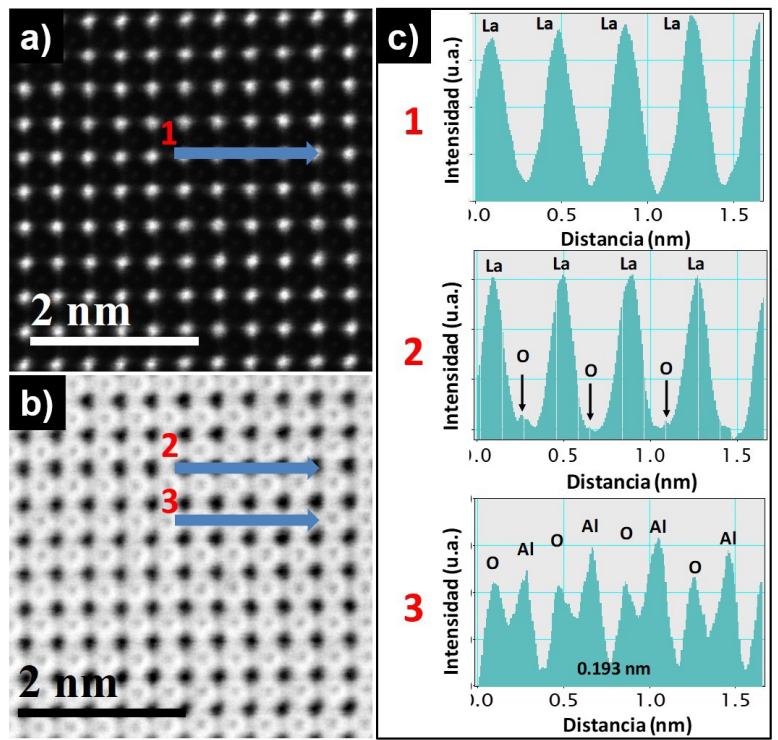

Fuente: Elaboración del autor. 
contribución del $\mathrm{Al}$ y $\mathrm{O}$, con una buena relación señal/ruido. Por consiguiente, la técnica $\mathrm{ABF}-\mathrm{STEM}$ mejora el contraste de las columnas atómicas de elementos ligeros y se puede emplear con éxito para detectar la posición de los elementos ligeros en los materiales.

La simulación de las imágenes ABF y HAADF-STEM se realizó utilizando el programa QSTEM (Koch, 2002) que utiliza el algoritmo de multicapas. Se encontró que las imágenes simuladas con las condiciones instrumentales concuerdan con las imágenes experimentales. Las figuras $5 \mathrm{a}$ y b muestran las imágenes simuladas de HAADF-STEM y ABF-STEM de la fase $\mathrm{LaAlO}_{3}$ vistas a lo largo de la orientación [001], respectivamente. De la figura 5a, las columnas atómicas correspondientes al La son claramente visibles. Un contraste débil entre estas columnas sugiere la ubicación de las columnas de $\mathrm{Al}$, pero no se resuelven claramente en esta imagen. En la imagen simulada ABFSTEM (figura $5 b$ ), todas las columnas visibles aparecen con contraste negativo. Como se menciona en la imagen experimental ABF-STEM (figura 4b), las columnas $\mathrm{La}, \mathrm{Al}$ y $\mathrm{O}$ se distinguen con una mejor intensidad de señal/ ruido. La comparación directa de las imágenes experimentales y simuladas de ABF-STEM se lleva a cabo mediante la comparación de los perfiles de intensidad determinados a través de las columnas atómicas La-O (figura $5 c$ ). La curva negra muestra el perfil de intensidad de la imagen experimental ABFSTEM, esto servirá como punto de referencia. La curva roja muestra el perfil de intensidad de la imagen simulada $\mathrm{ABF}-\mathrm{STEM}$ de la fase $\mathrm{LaAlO}_{3}$. El pico co-

Figura 5. a) y b) Imágenes simuladas de HAADF y ABF-STEM de la fase $\mathrm{LaAlO}_{3}$; c) perfiles de intensidad a través de las imágenes experimentales y simuladas de ABF-STEM.
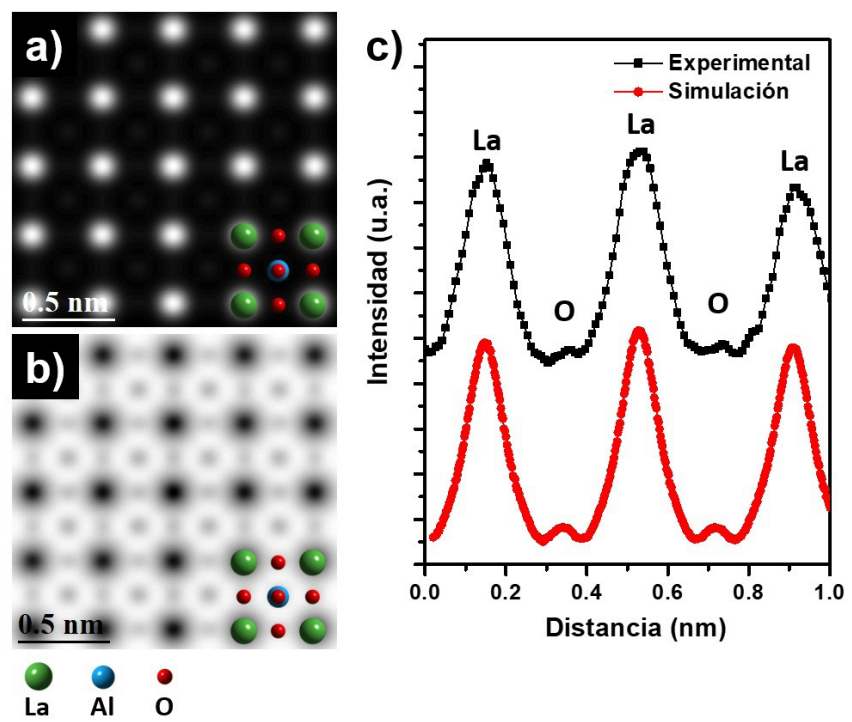

Fuente: Elaboración del autor. 
rrespondiente a La tiene la misma intensidad en comparación con la curva experimental; incluso la relación entre los picos de los perfiles de intensidades de La y O es consistente con la imagen experimental. De este modo, para obtener imágenes directas de la estructura atómica de una muestra, se pueden utilizar tanto HRTEM como STEM. Ambas brindan información en el espacio real, lo que facilita la visualización directa de la estructura y defectos cristalinos de los materiales. Sin embargo, debido a la sensibilidad de las imágenes HAADF-STEM y ABF-STEM al contraste por número atómico, hace que estas sean muy útiles para visualizar tanto elementos pesados como ligeros que en HRTEM convencional sería muy difícil.

\section{Conclusiones}

Se evaluó el rendimiento del STEM equipado con un corrector de Cs. Las aberraciones axiales de un sistema de lentes formadoras del haz de electrones son directamente visibles en un ronchigrama, por lo cual se considera como parte fundamental del procedimiento de alineación de los correctores de aberración disponibles en un STEM. La efectividad de la metodología de simulación propuesta demostró una comparación entre los ronchigramas experimentales y simulados obtenidos con los parámetros del instrumento con un ángulo medio superior a 40 mrad. Las transformadas rápidas de Fourier obtenidas de las imágenes de STEM de Si[110] tomadas con un voltaje de aceleración de $80 \mathrm{kV}$ muestran una resolución espacial de hasta $0.09 \mathrm{~nm}$. STEM con corrección de Cs visualiza con precisión la estructura atómica tanto de elementos ligeros como de pesados, obteniendo imágenes simultáneas de ABF-STEM y HAADF-STEM controlando los ángulos de recolección del detector circular BF y del detector anular ADF, respectivamente. Las condiciones experimentales para visualizar las columnas atómicas de la muestra $\mathrm{LaAlO}_{3}$ fueron para ABF 11-22 mrad y para HAADF con 90-170 mrad. Mediante la técnica ABF-STEM se demostró que se pueden detectar tanto elementos ligeros como pesados en las redes cristalinas, en este caso fue posible visualizar las columnas atómicas del $\mathrm{La}, \mathrm{Al}$ y $\mathrm{O}$ presentes en la muestra LAO. Las simulaciones de las imágenes de ABF-STEM y HAADF-STEM basadas en el método multicapas de Cowley y Moodie se han utilizado como herramienta para identificar el contraste de las diferentes columnas atómicas. ABF-STEM, es una técnica que mejora el contraste de las columnas atómicas y puede emplearse para la detección de elementos ligeros de diversos materiales.

\section{Referencias}

Barthel, J. (2007). http://www.er-c.org/barthel/drprobelight/

Bell, D. C., C. J. Russo y D. V. Kolmykov. (2012). $40 \mathrm{keV}$ atomic resolution TEM. Ultramicroscopy, 114: 31-37. https://doi.org/10.1016/j.ultramic.2011.12.001 
Benetatos, N. M., B. W. Smith, P. A. Heiney y K. I. Winey. (2005). Toward reconciling STEM and SAXS data from ionomers by investigating gold nanoparticles. Macromolecules, 38: 9251-9257. https://doi.org/10.1021/ma051419i

Cowley, J. M. (1986). Electron diffraction phenomena observed with a high resolution STEM instrument. Journal of Electron Microscopy Technique, 3: 25-44. https://doi.org/10.1002/jemt.1060030105

Cowley, J. M. y A. F. Moodie. (1957). The scattering of electrons by atoms and crystals. I. A new theoretical approach. Acta Crystallographica, 10: 609-619. https:// doi.org/10.1107/S0365110X57002194

Crewe, A. V., J. Wall y J. Langmore. (1970). Visibility of single atoms. Science, 168 (3937): 1338-1340. https://doi.org/10.1126/science.168.3937.1338

Dupouy, G. (2017). Chapter four - Electron microscopy at very high voltages. En Advances in Imaging and Electron Physics. Elsevier, 261-340.

Dwyer, C. (2010). Simulation of scanning transmission electron microscope images on desktop computers. Ultramicroscopy, 110(3):195-198. https://doi.org/10.1016/j. ultramic.2009.11.009

Esparza, R., A. F. García-Ruiz, J. J. Velázquez Salazar, R. Pérez y M. J. Yacamán. (2013). Structural characterization of Pt-Pd core-shell nanoparticles by Cs-corrected STEM. Journal of Nanoparticle Research, 15(1): 1342. https://doi.org/10.1007/ s11051-012-1342-2

Esparza, R., O. Téllez-Vázquez, G. Rodríguez-Ortiz, A. Ángeles-Pascual, S. Velumani y R. Pérez. (2014). Atomic structure characterization of Au-Pd bimetallic nanoparticles by aberration-corrected scanning transmission electron microscopy. The Journal of Physical Chemistry C, 118(38): 22383-22388. https://doi.org/10.1021/ jp507794z

Findlay, S. D., N. Shibata, H. Sawada, E. Okunishi, Y. Kondo, T. Yamamoto y Y. Ikuhara. (2009). Robust atomic resolution imaging of light elements using scanning transmission electron microscopy. Applied Physics Letters, 95: 191913. https://doi.org/10.1063/1.3265946

Garcia, A., A. M. Raya, M. M. Mariscal, R. Esparza, M. Herrera, S. I. Molina, G. Scavello, P. L. Galindo, M. J. Yacamán y A. Ponce. (2014). Analysis of electron beam damage of exfoliated MoS2 sheets and quantitative HAADF-STEM imaging. Ultramicroscopy, 146: 33-38. https://doi.org/10.1016/j.ultramic.2014.05.004

Geuens, P. y D. Van Dyck. (2003). About forbidden and weak reflections. Micron, 34: 167-171. https://doi.org/10.1016/S0968-4328(03)00032-5

Haider, M., S. Uhlemann y J. Zach. (2000). Upper limits for the residual aberrations of a high-resolution aberration-corrected STEM. Ultramicroscopy, 81(3-4): 163-175. https://doi.org/10.1016/S0304-3991(99)00194-1

Haider, M., P. Hartel, H. Müller, S. Uhlemann y J. Zach. (2009). Current and future aberration correctors for the improvement of resolution in electron microscopy. Philosophical Transactions of the Royal Society A, 367: 3665-3682. https:// doi.org/ 10.1098/rsta.2009.0121

Haider, M., S. Uhlemann, E. Schwan, H. Rose, B. Kabius y K. Urban. (1998). Electron microscopy image enhanced. Nature, 392(6678): 768-769. https://doi.org/10.1038/33823 
Hawkes, P. W. (2004). Advances in imaging and electron physics: Elsevier.

Huang, R., H. C. Ding, W. I Liang, Y. C. Gao, X. D. Tang, Q. He, C. G. Duan, Z. Zhu, J. Chu, C. A. J. Fisher, T. Hirayama, Y. Ikuhara y Y. H. Chu. (2014). Atomic scale visualization of polarization pinning and relaxation at coherent $\mathrm{BiFeO}_{3} / \mathrm{LaA}$ $1 \mathrm{O}_{3}$ interfaces. Advanced Functional Materials, 24(6): 793-799. https://doi. org/10.1002/adfm.201301470

Ishikawa, R., E. Okunishi, H. Sawada, Y. Kondo, F. Hosokawa y E. Abe. (2011). Direct imaging of hydrogen-atom columns in a crystal by annular bright-field electron microscopy. Nature Materials, 10(4): 278-281. https://doi.org/10.1038/ nmat2957

James, E. M. y N. D Browning. (1999). Practical aspects of atomic resolution imaging and analysis in STEM. Ultramicroscopy, 78: 125-139. https://doi.org/10.1016/ S0304-3991(99)00018-2

Jones, L., K. E. MacArthur, V. T. Fauske, A. T. J. van Helvoort y P. D. Nellist. (2014). Rapid estimation of catalyst nanoparticle morphology and atomic-coordination by high-resolution Z-contrast electron microscopy. Nano Letters, 14: 6336-6341. https://doi.org/10.1021/nl502762m

Kirkland, E. J., R. F. Loane y J. Silcox. (1987). Simulation of annular dark field stem images using a modified multislice method. Ultramicroscopy, 23: 77-96. https:// doi.org/10.1016/0304-3991(87)90229-4

Klenov, D. O., D. G. Schlom, H. Li y S. Stemmer. (2005). The interface between single crystalline (001) LaAlO3 and (001) silicon. Japanese Journal of Applied Physics, 44 (5L): L617. https://doi.org/10.1143/JJAP.44.L617

Klie, R. F. y Y. Zhu. (2005). Atomic resolution STEM analysis of defects and interfaces in ceramic materials. Micron, 36(3): 219-231. https://doi.org/10.1016/j. micron.2004.12.003

Koch, C. (2002). Determination of core structure periodicity and point defect density along dislocations. USA: Arizona State University.

Kotaka, Y. (2012). Direct visualization method of the atomic structure of light and heavy atoms with double-detector $\mathrm{Cs}$-corrected scanning transmission electron microscopy.Applied Physics Letters, 101(13):133107.https://doi.org/10.1063/1.4756783

Lee, P. W., V. N. Singh, G. Y. Guo, H. -J. Liu, J. -C. Lin, Y. -H. Chu, C. H. Chen y M. -W. $\mathrm{Chu}$. (2016). Hidden lattice instabilities as origin of the conductive interface between insulating $\mathrm{LaAlO}_{3}$ and $\mathrm{SrTiO}_{3}$. Nature Communications, 7: 12773 . https://doi.org/10.1038/ncomms12773

Maurice, J. L., C. Carrétéro, M. J. Casanove, K. Bouzehouane, S. Guyard, É. Larquet y J. P. Contour. (2006). Electronic conductivity and structural distortion at the interface between insulators $\mathrm{SrTiO}_{3}$ and $\mathrm{LaAlO}_{3}$. Physica Status Solidi, 203(9): 2209-2214. https://doi.org/10.1002/pssa.200566033

Mayoral, A., R. Esparza, F. L. Deepak, G. Casillas, S. Mejía-Rosales, A. Ponce y M. J. Yacamán. (2011). Study of nanoparticles at UTSA: one year of using the first JEM-ARM200F installed in USA. Jeol News, 46 (1): 1-5.

Mishra, R., R. Ishikawa, A. R. Lupini y S. J. Pennycook. (2017). Single-atom dynamics in scanning transmission electron microscopy. MRS Bulletin, 42(9): 644- 
652. https://doi.org/10.1557/mrs.2017.187

Okunishi, E., I. Ishikawa, H. Sawada, F. Hosokawa, M. Hori y Y. Kondo. (2009). Visualization of light elements at ultrahigh resolution by stem annular bright field microscopy. Microscopy and Microanalysis, 15(S2):164-165.https://doi.org/10.1017/ S1431927609093891

Pennycook, S. J., D. E. Jesson, A. J. McGibbon y P. D. Nellist. (1996). High angle dark field STEM for advanced materials. Microscopy, 45(1): 36-43. https://doi.org/10.1093/ oxfordjournals.jmicro.a023410

Rodenburg, J. M. y E. B. Macak. (2002). Optimising the resolution of TEM/STEM with the electron ronchigram. Microscopy and Analysis, 5-7.

Rodríguez-Proenza, C. A., J. P. Palomares-Báez, M. A. Chávez-Rojo, A. F. GarcíaRuiz, C. L. Azanza-Ricardo, A. Santoveña-Uribe, G. Luna-Bárcenas, J. L. Rodríguez-López y R. Esparza. (2018). Atomic surface segregation and structural characterization of PdPt bimetallic nanoparticles. Materials, 11(10): 1882. https://doi.org/10.3390/ma11101882

Sasaki, T., H. Sawada, F. Hosokawa, Y. Kohno, T. Tomita, T. Kaneyama, Y. Kondo, K. Kimoto, Y. Sato y K. Suenaga. (2010). Performance of low-voltage STEM/TEM with delta corrector and cold field emission gun. Journal of Electron Microscopy, 59(S1): S7-S13. https://doi.org/10.1093/jmicro/dfq027

Sawada, H., T. Sannomiya, F. Hosokawa, T. Nakamichi, T. Kaneyama, T. Tomita, Y. Kondo, T. Tanaka, Y. Oshima, Y. Tanishiro y K. Takayanagi. (2008). Measurement method of aberration from ronchigram by autocorrelation function. Ultramicroscopy, 108(11): 1467-1475. https://doi.org/10.1016/j.ultramic.2008.04.095

Spence, J. C. H. (2013). High-resolution electron microscopy. OUP Oxford.

Zhou, D., K. Müller-Caspary, W. Sigle, F. F. Krause, A. Rosenauer y P. A. van Aken. (2016). Sample tilt effects on atom column position determination in ABF-STEM imaging. Ultramicroscopy, 160: 110-117. https://doi.org/10.1016/j.ultramic.2015.10.008 